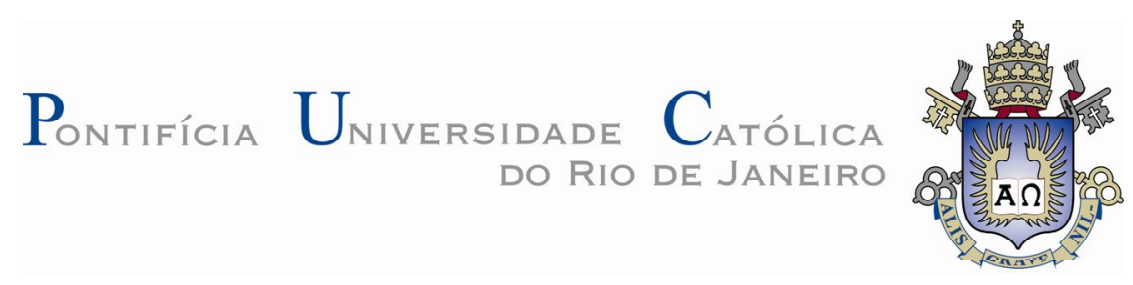

David Iván Maldonado Távara

\title{
Estudo Numérico de Jato Circular Espiralado Incidente em uma Placa Aquecida
}

Tese de Doutorado

Tese apresentada como requisito parcial para obtenção do título de Doutor pelo Programa de PósGraduação em Engenharia Mecânica da PUC-Rio.

Orientadora: Angela Ourivio Nieckele

Rio de Janeiro

Fevereiro de 2011 


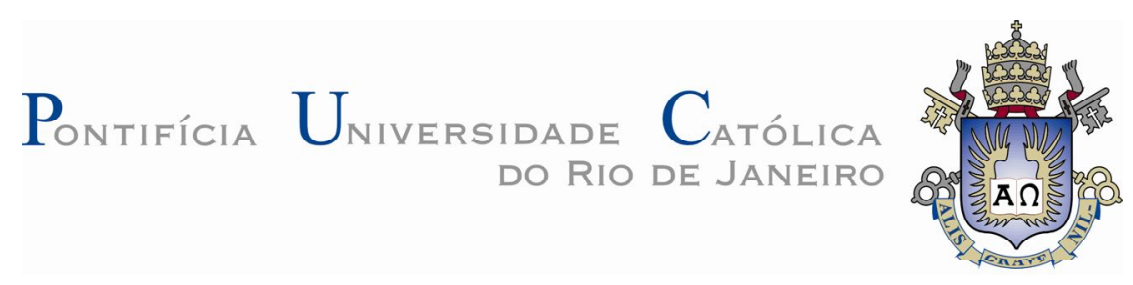

\title{
David Iván Maldonado Távara
}

\section{Estudo Numérico de Jato Circular Espiralado Incidente em uma Placa Aquecida}

Tese apresentada como requisito parcial para obtenção do título de Doutor pelo Programa de Pós-Graduação em Engenharia Mecânica do Centro Técnico Científico da PUC-Rio. Aprovada pela Comissão Examinadora abaixo assinada.

\author{
Profa. Angela Ourivio Nieckele \\ Orientadora \\ Departamento de Engenharia Mecânica - PUC-Rio \\ Prof. Sergio Leal Braga \\ Departamento de Engenharia Mecânica - PUC-Rio \\ Profa. Mônica Feijó Naccache \\ Departamento de Engenharia Mecânica - PUC-Rio
}

Prof. Luiz Eduardo Bittencourt Sampaio Universidade Federal Fluminense - UFF

Prof. Roney Leon Thompson Universidade Federal Fluminense - UFF

Prof. José Eugenio Leal Coordenador Setorial do Centro Técnico Científico - PUC-Rio 
Todos os direitos reservados. É proibida a reprodução total ou parcial do trabalho sem autorização da universidade, do autor e do orientador.

\section{David Ivan Maldonado Távara}

Graduou-se em Engenharia Mecânica na Universidad Nacional de Trujillo, Trujillo - La Libertad - Peru

Mestrado em Engenharia Mecânica, pela Pontifícia Universidade Católica de Rio de Janeiro- PUC-Rio, Rio de Janeiro - Brasil.

Ficha Catalográfica

Maldonado Távara, David Iván

Estudo numérico de jato circular espiralado incidente em uma placa aquecida / David Iván Maldonado Távara ; orientador: Angela Ourivio Nieckele. - 2011.

211 f. : il. (color.) ; $30 \mathrm{~cm}$

Tese (doutorado)-Pontifícia Universidade Católica do Rio de Janeiro, Departamento de Engenharia Mecânica, 2011.

Inclui bibliografia

1. Engenharia mecânica - Teses. 2. Jato circular espiralado incidente. 3. Turbulência. 4. RANS. 5. LES. 6. Troca térmica. I. Nieckele, Angela Ourivio. II. Pontifícia Universidade Católica do Rio de Janeiro. Departamento de Engenharia Mecânica. III. Título. 


\title{
Agradecimentos
}

\author{
À Professora Angela Ourivio Nieckele, pela amizade e paciência para \\ transmitir seu conhecimento, pela incansável motivação na realização \\ deste trabalho.
}
À CAPES e a PUC-Rio pelos auxílios concedidos, sem os quais este trabalho não teria sido possível.

\begin{abstract}
Aos meus amigos e colegas de Termociências e do sexto andar de pós mecânica, por compartir comigo este caminho e sempre estarmos dispostos a nos ajudar um dos outros mesmo nos momentos difíceis.
\end{abstract}


Dedico esta teses a meus pais Germán e Luz pelo apoio incondicional em toda minha vida. 


\section{Resumo}

Távara, David Iván Maldonado; Nieckele, Angela Ourivio. Estudo Numérico de Jato Circular Espiralado Incidente em uma Placa Aquecida. Rio de Janeiro, 2011. 211p. Tese de Doutorado Departamento de Engenharia Mecânica, Pontifícia Universidade Católica do Rio de Janeiro.

Escoamentos espiralados incidentes em superfícies são escoamentos altamente complexos, envolvendo uma variedade grande de regimes. A previsão numérica deste tipo de escoamento pode auxiliar na compreensão dos diversos fenômenos envolvidos, o que poderá permitir o desenvolvimento de processos mais eficientes, assim como auxiliar na avaliação de modelos de turbulência.

Visando avaliar o desempenho de diferentes modelos de turbulência para a previsão de um jato axi-simétrico espiralado incidindo em uma placa quente, determinou-se numericamente o escoamento com o auxílio da ferramenta computacional FLUENT, utilizando duas metodologias. A primeira baseada na média de Reynolds (RANS) e a segunda na simulação de grandes escalas (LES).

Diversos modelos RANS de duas equações diferenciais foram testados, visando identificar a validade de aplicação de cada um deles através da comparação com dados experimentais disponíveis. A metodologia LES foi avaliada utilizando o modelo dinâmico de sub-malha de Smagorinsky.

Apesar do alto custo, devido à necessidade de utilizar grande espaço de memória e tempo de simulação, os resultados obtidos com a metodologia LES foram significativamente superiores, uma vez que o escoamento apresenta alta anisotropia, a qual os modelos RANS apresentam dificuldade em prever.

\section{Palavras-chave} Térmica.

Jato Circular Espiralado Incidente, Turbulência, RANS, LES, Troca 


\section{Abstract}

Távara, David Iván Maldonado; Nieckele, Angela Ourivio (Advisor). Numerical Study of Swirling Round Jet Impinging on a Heated Plate. Rio de Janeiro, 2011. 211p. DSc. Thesis - Departamento de Engenharia Mecânica, Pontifícia Universidade Católica do Rio de Janeiro.

Incident swirling jets are complex flow, which involve a large variety of regimes. The numerical prediction of this type of flow can help understand the several phenomena present, which could allow development of more efficient processes, as well as assist in the evaluation of turbulence models.

Aiming to evaluate the performance of different turbulence models in the predict of an axi-symmetric swirling jet impinging to a hot plate, the flow field was determined with the computational tool FLUENT, by employing two methodologies. The first one is based on the Reynolds average (RANS) and the second one is based on the Large Eddy Simulation (LES).

Several two differential equations RANS models were tested, in order to identify their range of validity, by comparing with available experimental data. The methodology LES was evaluated with the dynamic Smagorinsky sub-grid model.

In spite of the high cost, due to the need of utilizing a large memory space and simulation time, the results obtained with the LES methodology were significantly superior, due to the high anisotropy of the flow, which the RANS models have difficult to predict.

\section{Keywords}

Swirling Round Jet Impinging; Turbulence, RANS, LES, Heat Transfer. 


\section{Lista de Símbolos}

\begin{tabular}{|c|c|}
\hline$a$ & Variável original antes da Filtragem \\
\hline $\bar{a}$ & Variável filtrada \\
\hline$A_{f}=S_{f}$ & Vetor área da face $f$ do volume de controle $i$ \\
\hline$c_{p}$ & Calor especifico a pressão constante \\
\hline$C_{k}$ & Constante de Kolmogorov \\
\hline $\mathrm{C}_{\mathrm{p}}$ & Calor especifico \\
\hline$C_{s}$ & Coeficiente de Smagorinsky \\
\hline $\mathrm{D}$ & Diâmetro do jato \\
\hline$E(k)$ & Energia Cinética Turbulenta \\
\hline$G_{\Delta_{C}}$ & Filtro de Kernel \\
\hline $\mathrm{H}$ & Distancia entre o bocal na saída do jato e a parede \\
\hline$y+$ & Distancia adimensional para parede \\
\hline$\kappa$ & Energia Cinética Turbulenta \\
\hline $\bar{k}$ & valor médio do campo de energia cinética turbulenta \\
\hline$k$ & Comprimento de onda \\
\hline k & Condutividade térmica \\
\hline$l_{d}$ & Comprimento do turbilhão em pequenas escalas \\
\hline$\ell_{s}$ & O comprimento de escala de Smagorinsky \\
\hline$l_{c}$ & Comprimento característico \\
\hline$L$ & Comprimento do turbilhão em grandes escalas \\
\hline$\dot{m}_{f}$ & Fluxo de massa cruzando a face $f$ \\
\hline$N_{i}$ & Número de faces do i-ésimo Volume de Controle \\
\hline$N g l$ & Número de graus de liberdade \\
\hline $\mathrm{N}-\mathrm{S}$ & Equações de Navier Stokes \\
\hline
\end{tabular}




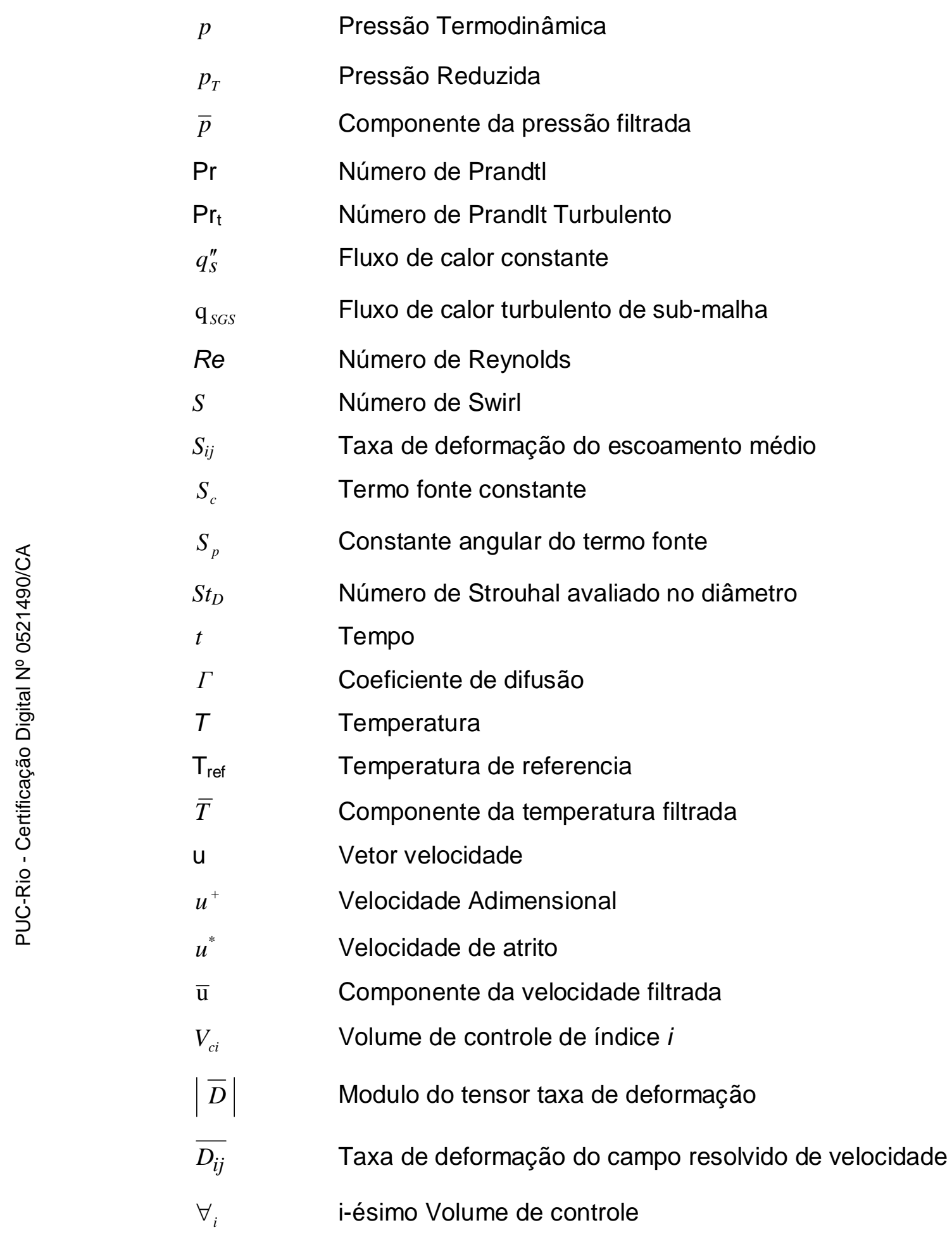

\section{Símbolos gregos}

$\begin{array}{ll}\rho & \text { Massa específica ou densidade }\left(\mathrm{kg} / \mathrm{m}^{3}\right) \\ \mu & \text { Viscosidade absoluta }\end{array}$




$\begin{array}{ll}v_{t} & \text { Viscosidade turbulenta } \\ v_{t, S G S} & \text { Viscosidade turbulenta sub-malha } \\ \varepsilon & \text { Taxa de dissipação de energia } \\ \omega & \text { Taxa de dissipação especifica } \\ \alpha & \text { Difusividade térmica } \\ \Gamma & \text { Coeficiente de difusão } \\ \Delta & \text { Largura de filtro espacial ou temporal } \\ \Delta t & \text { Passo de tempo } \\ \Omega_{i j} & \text { Taxa de rotação do escoamento médio } \\ \mu_{S G S} & \text { Viscosidade turbulenta da sub-malha } \\ \tau_{w} & \text { Tensão cisalhante na parede } \\ \tau_{i j} & \text { Tensor de Reynolds sub-malha } \\ \phi & \text { Campo escalar genérico } \\ \bar{\phi} & \text { Média de uma Grandeza } \\ \phi^{\prime} & \text { Valor instantâneo } \\ \phi_{f} & \text { Valor de } \phi \text { na face }\end{array}$




\section{Sumário}

1 Introdução 21

1.1 Objetivo e Escopo do Trabalho 27

$\begin{array}{ll}1.2 \text { Organização do Trabalho } & 29\end{array}$

2 Revisão Bibliográfica 30

2.1 Física do Problema 30

2.1.1 Jato Livre 33

2.1.2 Jato de Parede $\quad 35$

2.1.3 Jatos Incidentes $\quad 36$

2.1.4 Características Dinâmicas do Jato incidente 38

2.1.5 Transferência de Calor em Jatos Incidentes 41

2.1.6 Jatos Incidentes Espiralados $\quad 45$

2.2 Turbulência e seus Respectivos Modelos de Simulação 49

2.3 Adaptação dos Modelos de Turbulência ao Fenômeno do jato Incidente 51

3 Modelamento Matemático 53

3.1 Equações Governantes $\quad 57$

3.2 Equações de Médias de Reynolds - RANS 59

3.2.1 Modelo $\kappa-\varepsilon$ padrão $\quad 62$

3.2.2 Modelo $\kappa-\varepsilon$ Realizável 63

3.2.3 Modelo $\kappa-\varepsilon$ RNG 66

3.2.4 Modelo $\kappa-\omega$ SST $\quad 67$

3.2.5 Modelo do Tensor de Reynolds, RSM 70

3.2.6 Modelos RANS nas regiões próximas às paredes 76 
3.3 Simulação de Grandes Escalas - LES 80

3.3.1 Modelo sub-malha de Smagorinsky 85

3.3.2 Modelo de Smagorinsky Dinâmico 86

4 Método Numérico 90

4.1 Discretização da Equação Geral de Transporte 91

4.1.1 Esquema Power-Law 93

4.1.2 Esquema Diferenças Centrais 94

4.1.3 Esquema QUICK 97

4.1.4 Discretização Temporal 97

4.2 Discretização das Equações de Navier-Stokes 98

4.2.1 Acoplamento pressão-velocidade 100

4.3 Metodologia de Solução do Sistema Algébrico 101

5 Resultados 104

5.1 Experimento 105

5.2 Domínio Computacional 107

5.3 Condições de Contorno 108

5.4 Modelos RANS 110

5.4.1 Modelos RANS, Caso $S=0$

5.4.1.1 Velocidade Radial Média, Caso $S=0$

5.4.1.2 Estatísticas de Segunda Ordem, Caso $S=0$

5.4.1.3 Coeficiente de Atrito e de Pressão, Caso $S=0$

5.4.1.4 Temperatura, Caso $S=0 \quad 127$

5.4.1.5 Fluxo de Calor Turbulento, Caso $S=0 \quad 131$

5.4.1.6 Número de Nusselt, Caso $S=0$

5.4.1.7 Influência da Energia Cinética do Jato, Caso $S=0$

5.4.2 Modelos RANS, Caso $S=0,3$

5.4.2.1 Velocidade Radial Média, Caso $S=0,3$

5.4.2.2 Estatísticas de Segunda Ordem, Caso $S=0,3$ 
5.4.2.3 Temperatura, Caso $S=0,3 \quad 148$

5.4.2.4 Fluxo de Calor Turbulento, Caso $S=0,3$

5.4.2.5 Número de Nusselt, Caso $S=0,3$

5.4.2.6 Influência da Energia Cinética do Jato, Caso $S=0,3$

5.4.3 Modelos RANS, Caso $S=0,5$

5.4.3.1 Velocidade Radial Média, Caso $S=0,5$

5.4.3.2 Estatísticas de Segunda Ordem, Caso $S=0,5$

5.4.3.3 Temperatura, Caso $S=0,5$

5.4.3.4 Fluxo de Calor Turbulento, Caso $S=0,5$

5.4.3.5 Número de Nusselt, Caso $S=0,5 \quad 166$

5.4.3.6 Influência da Energia Cinética do Jato, Caso $S=0,5$

5.5 Simulação de Grandes Escalas (LES) 170

5.5.1 Velocidade Radial Média, Caso LES, $S=0$

5.5.2 Estatísticas de Segunda Ordem, LES, $S=0$

5.5.3 Número de Nusselt, LES, $S=0 \quad 179$

6 Comentários Finais e Conclusões 182

6.1 Conclusões 183

6.2 Recomendações 186

Referências Bibliográficas $\quad 187$

APÊNDICE A Domínio Computacional e Malha 198

A.1 Simulações RANS 198

A.1.1 Teste de malha para Simulações RANS, $S=0 \quad 200$

A.1.2 Teste de malha para Simulações RANS, $S=0,5$

A.1.3 Teste de malha para Simulações RANS, S=0,5 205

A.2 Simulações LES 207

APÊNDICE B Teste para tamanho do Domínio 210 


\section{Lista de Figuras}

Figura 1.1- Representação esquemática de um jato incidindo sobre uma superfície plana.

Figura 1.2- Hipóteses esquemática de equilíbrio de Kolmogorov (Pope, 2000).

Figura 2.1- Visualização do escoamento de um jato incidente (Lee e Lee,2000).

Figura 2.2- Esquema da visualização de Leonardo da Vinci.

Figura 3.1- Grandes vórtices na camada limite turbulenta (Corrsin e Kistler, 1954).

Figura 3.2- Comparação entre as diferentes modelagens da turbulência.

Figura 4.1- Volume de controle tipo, na discretização da equação de transporte.

Figura 4.2- Volume de controle unidimensional.

Figura 4.3- Diagrama das variáveis normalizadas, NVD (Choi et. al., 1995).

Figura 4.4- Seqüência de malhas para o esquema Multigrid.

Figura 5.1- Vista frontal da geometria utilizada por Abrantes (2005).

Figura 5.2- $\quad$ Estações de medição sobre a superfície, Abrantes (2005).

Figura 5.3- Domínio computacional com as condições de contorno e estações de medição empregadas.

Figura 5.4- Perfis de velocidade média e energia cinética turbulenta do jato. Caso $S=0$.

Figura 5.5- Perfis da dissipação especifica $\omega$, e a taxa de dissipação $\varepsilon$ do jato. Caso $S=0$.

Figura 5.6- $\quad$ Perfis de velocidade média axial e radial do jato. 
Figura 5.7- Perfis de velocidade média axial e radial do jato.

Caso $S=0,5 \quad 110$

Figura 5.8- Velocidade radial média. Caso $S=0$. Cinco modelos. 113

Figura 5.9- Perfis de velocidade radial média. Caso $S=0$. $\kappa-\omega$ STT (LRC) e RSM (EWT).

Figura 5.10- Perfil de velocidade média em unidades de parede. Caso $S=0$. 116

Figura 5.11- Velocidades turbulentas radial. Caso $S=0$. Cinco modelos.

Figura 5.12- Velocidades turbulentas axial. Caso $S=0$. Cinco modelos.

Figura 5.13- Tensão cisalhante de Reynolds turbulento. Caso $S=0$. Cinco modelos.

Figura 5.14- Perfis de velocidade radial turbulenta, Caso $S=0$. $\kappa-\omega$ STT e RSM (EWT).

Figura 5.15- Perfis de velocidade axial turbulenta, Caso $S=0$. $\kappa-\omega$ STT (LRC) e RSM (EWT).

Figura 5.16- Perfis da tensão cisalhante de Reynolds turbulento, Caso $S=0 . \kappa-\omega S T T$ (LRC) e RSM (EWT).

Figura 5.17- Energia cinética turbulenta, $y / D=0,02$. Caso $S=0$. Cinco modelos.

Figura 5.18- Coeficiente de atrito ao longo da superfície. Caso $S=0$. Cinco modelos.

Figura 5.19- Coeficiente de pressão na superfície. Caso $S=0$. Cinco modelos.

Figura 5.20- Temperatura adimensional nas estações radiais $r / D$. Caso $S=0$.

Figura 5.21- Temperatura em coordenadas axiais $(y / D)$, Caso $S=0$.

Figura 5.22- Perfil de Temperatura média em unidades de parede. Caso $S=0$.

Figura 5.23- Perfil do fluxo de calor turbulento em unidades de 
parede. Caso $S=0$.

Figura 5.24- Número de Nusselt na superfície. Caso $S=0$. Cinco modelos.

Figura 5.25- Número de Nusselt na superfície. Caso $S=0$. $\kappa-\omega$ STT e RSM (EWT).

Figura 5.26- Influência do Prandtl turbulento no número de Nusselt. Modelo $\kappa-\omega$ SST LRC. Caso $S=0$.

Figura 5.27- Influência do perfil da energia cinética turbulenta do jato no número de Nusselt. Modelo $\kappa-\omega$ SST LRC. Caso $S=0$.

Figura 5.28- Influência do perfil da energia cinética turbulenta do jato nas tensões de Reynolds. Modelo $\kappa-\omega$ SST LRC. Caso $\mathrm{S}=0$.

Figura 5.29- Influência do perfil da energia cinética turbulenta do jato no número de Nusselt. Modelo $\kappa-\omega$ SST LRC. Caso $S=0$.

Figura 5.30- Velocidade radial média. Caso $S=0,3$. Quatro modelos.

Figura 5.31- Perfis de velocidade radial média. Caso $S=0,3$. $\kappa-\omega$ STT (LRC) e RSM (EWT).

Figura 5.32- $\quad$ Perfil de velocidade média em unidades de parede. Caso $S=0,3$.

Figura 5.33- Velocidades turbulentas radial. Caso $S=0,3$. Quatro modelos.

Figura 5.34 Velocidades turbulentas axial. Caso $S=0,3$. Quatro modelos.

Figura 5.35- Perfis de velocidade radial turbulenta.

Caso $=0,3$.

Figura 5.36- Perfis de velocidade axial turbulenta.

Caso $S=0,3$.

Figura 5.37- Energia cinética turbulenta, $y / D=0,02$. Caso $S=0,3$. Quatro modelos.

Figura 5.38- Temperatura adimensional nas estações radiais. 
Figura 5.39- Perfil de Temperatura média em unidades de parede, Caso $S=0,3$.

Figura 5.40- Perfil do fluxo de calor turbulento em unidades de parede, Caso $S=0,3$.

Figura 5.41- Número de Nusselt na superfície, Caso $S=0,3$. Quatro modelos.

Figura 5.42- Influência do perfil da energia cinética turbulenta do jato na velocidade radial média. Modelo $\kappa-\omega S S T$ LRC, Caso $S=0,3$.

Figura 5.43- Influência do perfil da energia cinética turbulenta do jato nas tensões de Reynolds. Modelo $\kappa-\omega$ SST LRC, 153 Caso $S=0,3$.

Figura 5.44- Influência do perfil da energia cinética turbulenta do jato no número de Nusselt. Modelo $\kappa-\omega$ SST LRC, 154 Caso $S=0,3$.

Figura 5.45- Velocidade radial média. Caso $S=0,5$. Quatro modelos.

Figura 5.46- Perfis de Velocidade Radial Média, Caso $S=0,5$. $\kappa-\omega S T T$ (LRC) e RSM (EWT).

Figura 5.47- Perfil de velocidade média em unidades de parede. Caso $S=0,5$.

Figura 5.48- Velocidades turbulentas radial. Caso $S=0,5$. Quatro modelos.

Figura 5.49- Velocidades turbulentas axial. Caso $S=0,5$. Quatro modelos.

Figura 5.50- Perfis de Velocidade Radial Turbulenta, Caso $S=0,5$.

Figura 5.51- Perfis de Velocidade Axial Turbulenta, Caso $S=0,5$.

Figura 5.52- Energia cinética turbulenta, $y / D=0,02$. Caso $S=0,5$. Quatro modelos.

Figura 5.53- Temperatura adimensional nas estações axiais, Caso 
$S=0,5$.

Figura 5.54- Perfil de Temperatura média em unidades de parede, Caso $S=0,5$. 165

Figura 5.55- Perfil do fluxo de calor turbulento em unidades de parede, Caso $S=0,5$.

Figura 5.56- Número de Nusselt na superfície, Caso $S=0,5$. Quatro modelos.

Figura 5.57- Influência do perfil da energia cinética turbulenta do jato na velocidade radial média. Modelo $\kappa-\omega$ SST LRC, Caso $S=0,5$.

Figura 5.58- Influência do perfil da energia cinética turbulenta do jato nas tensões de Reynolds. Modelo $\kappa-\omega$ SST LRC, Caso $S=0,5$.

Figura 5.59- Influência do perfil da energia cinética turbulenta do jato no número de Nusselt. Modelo $\kappa-\omega$ SST LRC, Caso $S=0,5$.

Figura 5.60- Evolução da velocidade no tempo - sondas P1, P2, P3, P4 e P5.

Figura 5.61- Perfis de velocidade radial média,

LES - Dinâmico.

Figura 5.62- Perfis de velocidade turbulenta em,

$r / D=0,5$

Figura 5.63- Perfis de velocidade radial turbulenta, LES Dinâmico.

Figura 5.64- Perfis de Velocidade Axial Turbulenta, LES Dinâmico.

Figura 5.65- Perfis do Tensor Cisalhante de Reynolds Turbulento, LES - Dinâmico.

Figura 5.66- Número de Nusselt na superfície, LES - Dinâmico.

Figura A.1- Detalhes do domínio computacional utilizado, malha 120x150.

Figura A.2- Teste de malha radial para $S=0$ através da 
Velocidade radial.

Figura A.3- Teste de malha radial para $\mathrm{S}=0$ através do $\mathrm{y}^{+}$e 0 Coeficiente de pressão $\left(C_{p}\right)$ na parede. 201

Figura A.4- Teste de malha radial para $S=0$ através Número de Nusselt $(\mathrm{Nu})$ e Coeficiente de Atrito $\left(\mathrm{C}_{\mathrm{f}}\right)$ na parede. 201

Figura A.5- Teste de malha axial para $\mathrm{S}=0$ através da Velocidade radial. 201

Figura A.6- $\quad$ Teste de malha axial para $S=0$ através do $y^{+}$e o Coeficiente de pressão (Cp) na parede. 202

Figura A.7- Teste de malha axial para $S=0$ através Número de Nusselt $(\mathrm{Nu})$ e Coeficiente de Atrito $\left(\mathrm{C}_{\mathrm{f}}\right)$ na parede. 202

Figura A.8- Teste de malha radial para $S=0,3$ através da Velocidade radial. 203

Figura A.9- Teste de malha radial para $S=0,3$ através Número de Nusselt (Nu) na parede. 203

Figura A.10- Teste de malha axial para $S=0,3$ através da Velocidade radial. 204

Figura A.11- Teste de malha axial para $S=0,3$ através Número de Nusselt (Nu) na parede. 204

Figura A.12- Teste de malha radial para $S=0,5$ através da Velocidade radial. 205

Figura A.13- Teste de malha radial para $\mathrm{S}=0,5$ através Número de Nusselt (Nu) na parede. 205

Figura A.14- Teste de malha axial para $S=0,5$ através da Velocidade radial. 206

Figura A.15- Teste de malha axial para $S=0,5$ através Número de Nusselt (Nu) na parede. 206

Figura A.16- Detalhe da malha utilizada na avaliação do modelo LES. 208

Figura A.17- Comparação entre as malhas 3,6 M e 5,4 M. 209

Figura B.1- Domínio computacional (D2). 210

Figura B.2- Resultados de teste do domínio computacional. 211 


\section{Lista de Tabelas}

Tabela 5.1- Posição das estações de medição dos perfis de velocidade e grandezas turbulentas

Tabela 5.2- Propriedades do Ar na condição de entrada. 110

Tabela 5.3- Coordenadas das sondas na Simulação de Grandes Escalas

Tabela A.1- Malhas escolhidas para a realização do teste de malha Simulações RANS.

Tabela A.2- Malhas escolhidas para a realização do teste de malha Simulações LES. 